\title{
Satisfação dos usuários na utilização de serviços públicos e privados de saúde em itinerários terapêuticos no sul do Brasil
}

\author{
Maristela Chitto Sisson ${ }^{1}$ \\ Maria Conceição de Oliveira² \\ Eleonor Minho Conill ${ }^{3}$ \\ Denise Pires ${ }^{4}$ \\ Antonio Fernando Boing 5 \\ Hosanna Pattrig Fertonani ${ }^{6}$
}

SISSON, M.C. et al. Users' satisfaction with the use of public and private health services within therapeutic Itineraries in southern Brazil. Interface - Comunic., Saude, Educ., v.15, n.36, p.123-36, jan./mar. 2011.

This study dealt with people's satisfaction with the itinerary taken when they use the public and private health systems in three state capitals in southern Brazil, in situations of health-disease-care. Taking a qualitative approach, 131 users of health insurance plans who received care for acute myocardial infarction, alcoholism, breast cancer and childbirth were interviewed. The itineraries consisted of great numbers of routes and arrangements among healthcare subsystems, particularly in the fields of mental health and oncology. The category "satisfaction" showed favorable evaluations in relation to acute myocardial infarction, breast cancer and childbirth because there was fast access to the appropriate technical resources. There was dissatisfaction relating to difficulties in information availability, installations, costs, coverage and authorizations for procedures. The study showed the importance of taking into consideration the plurality of the care systems and the subjectiveness of value judgments regarding the implementation of healthcare policies and programs.

Keywords: Health evaluation. Patient satisfaction. Health services.

Supplemental healthcare.
O estudo trata da satisfação das pessoas nos itinerários percorridos quando da utilização de serviços de saúde públicos e privados em três capitais do sul do Brasil em face de situações de saúde-doençacuidados. Trabalhando com abordagem qualitativa, foram entrevistados 131 usuários de planos de saúde que receberam cuidados em infarto agudo do miocárdio, alcoolismo, câncer de mama e parto. Em itinerários dotados de inúmeras trajetórias e arranjos entre subsistemas de serviços, sobretudo no campo da saúde mental e da oncologia, a categoria satisfação mostrou boa avaliação no infarto agudo do miocárdio, câncer de mama e parto, pela presença de acesso rápido a recursos técnicos adequados. Insatisfações referiram-se a dificuldades nas informações, instalações, custos, coberturas e autorizações de procedimentos. O estudo mostrou a importância de se considerar a pluralidade dos sistemas de cuidados e a subjetividade do julgamento de valor na implementação de políticas e programas de saúde.

Palavras-chave: Avaliação em saúde. Satisfação do paciente. Serviços de saúde. Saúde suplementar.

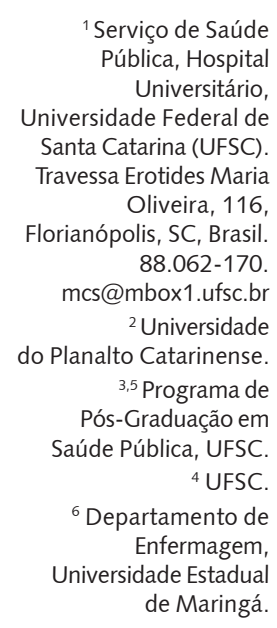




\section{Introdução}

A perspectiva dos usuários no desenvolvimento de estratégias de planejamento, gestão e controle de instituições prestadoras de serviços de saúde é reconhecida como fundamental nas esferas pública e privada do setor. Nesse sentido, estudos que avaliam a satisfação dos usuários são considerados estratégicos por permitirem sua intervenção em planos e programas, com o intuito de influenciar o nível de qualidade dos serviços prestados.

O interesse pela qualidade dos serviços de saúde está presente no Sistema Único de Saúde (SUS) e tem, gradativamente, tomado parte do cotidiano das organizações, suscitando relevante debate. No interior deste debate, cresce, potencialmente, o papel desempenhado pelos usuários, com fins de possibilitar um monitoramento técnico e social dos serviços e programas prestados à sociedade, seja na implementação de políticas públicas, seja na regulação das relações público-privadas do setor saúde.

Da mesma forma, no campo privado, a pesquisa sobre satisfação dos clientes dos planos de saúde é apontada como tarefa fundamental para a gestão das empresas e é utilizada como uma das dimensões que compõem os Índices de Desempenho na Saúde Suplementar (Silva Jr. et al., 2008).

Nos últimos anos, a Agência Nacional de Saúde Suplementar/ANS implementou um conjunto de medidas destinadas a redirecionar as ações de regulação, de um controle da saúde financeira das empresas para uma melhor qualificação da prestação e utilização dos serviços no setor. Neste contexto, tem utilizado, além dos estudos epidemiológicos convencionais, enfoques microssociais, por meio da perspectiva ou do mapeamento da experiência dos usuários dos serviços.

Nesta perspectiva, este estudo, como parte de uma pesquisa maior que analisou os percursos de usuários do subsistema de assistência médica suplementar em três capitais do sul do país (Porto Alegre, Florianópolis e Curitiba), em face da experiência de saúde-doença-cuidados (Conill et al., 2008), teve como objetivo conhecer e analisar sua satisfação em relação aos serviços e cuidados oferecidos e ao plano de saúde.

\section{A satisfação dos usuários e a avaliação de serviços}

Pesquisas de satisfação de pacientes têm crescentemente sido realizadas em serviços de saúde, oportunizando a revisão de uma larga faixa de componentes práticos para benefício potencial de procedimentos profissionais (Parker et al., 1996). Sua aplicação e utilidade evoluíram de um enfoque gerencial "de amenidades" à avaliação de pontos de vista de pacientes em várias questões, como a necessidade de informação nos componentes organizacionais e interpessoais do cuidado e até no valor do tratamento médico (Fitzpatrick, 1991). Além de realizar avaliações por demanda governamental, institucional ou corporativa, o autor oferece três razões pelas quais os profissionais de saúde devem, seriamente, ter em conta satisfação como medida: primeiro, porque existem evidências de que satisfação é uma importante medida sobre resultados e estado de saúde; segundo, porque permite que sejam avaliados componentes importantes de comunicação entre equipe de profissionais e usuários; e, terceiro, porque a avaliação da satisfação do paciente permite métodos alternativos de exame da provisão de cuidados de saúde.

Satisfação do paciente ou do usuário pode ser definida como as avaliações positivas individuais de distintas dimensões do cuidado à saúde (Linder-Pelz, 1982); e existem vários modelos para realizar esta medida, mas todos têm, como características comuns, as percepções do paciente sobre suas expectativas, valores e desejos (De Silva, 1999; Williams, 1994; Linder- Pelz, 1982).

Autores, como Donabedian (1980) e Pascoe (1983), consideram que a satisfação pode ser vista pela reação que os usuários têm diante do contexto, do processo e do resultado global de sua experiência relativa a um serviço.

Para Donabedian (1984), autor clássico da área da avaliação em saúde, a noção de satisfação do paciente é um dos elementos da avaliação da qualidade em saúde a ser complementada com a avaliação do médico e a da comunidade. Reforçando este postulado, Favaro e Ferris (1991) mostraram que a perspectiva do usuário, abordada por meio da sua satisfação, implica um julgamento sobre as características dos serviços e fornece informação essencial para completar e equilibrar a qualidade da atenção. 
Da forma como foi desenvolvido por Donabedian, o conceito de qualidade permitiu avançar no sentido de incorporar uma participação leiga - a dos pacientes - na definição de padrões e nas medidas da qualidade dos serviços. Consequentemente, observou-se um protagonismo cada vez maior dos usuários, e a ideia de satisfação do paciente como um atributo da qualidade tornou-se um objetivo em si e não somente uma garantia de continuidade do tratamento ou um meio de interferir na sua eficácia por uma maior adesão a ele, como frequentemente fora abordada (Williams, 1994; Vuori, 1987; Berger et al., 1989).

Gradativamente, um variado conjunto de pesquisas tomou como objeto de estudo a "satisfação do usuário", visando conhecer a opinião dos consumidores de serviços de saúde. Foi a partir desse momento que os estudos de avaliação em saúde passaram a utilizar o termo "usuário" (Vaitsman, Andrade, 2005).

De um modo geral, fatores relacionados à satisfação incluem: características sociodemográficas, status físico e psicológico; atitudes e expectativas sobre a estrutura, o processo e resultados do cuidado (Aharony, Strasse, 1993).

Não há consenso sobre quais resultados são mais fortes para influenciar a satisfação. Diferentes autores indicam relações com o tipo de cuidado oferecido e com o contexto no qual a satisfação do paciente é estudada, mas não se encontrou ainda uma correlação simples e direta entre satisfação e melhoria nos resultados. Pacientes satisfeitos parecem mais permeáveis em aderir aos tratamentos, embora a satisfação também possa ser vista como um antecedente causal do comportamento de melhora (Aharony, Strasse, 1993).

Um trabalho recente feito por Gerschman et al. (2007) avaliou a satisfação de beneficiários de planos de saúde de hospitais filantrópicos nos estados de São Paulo, Rio de Janeiro e Minas Gerais por intermédio do uso de grupos focais. As razões referidas para satisfação foram: o acesso, a hotelaria e a privacidade percebida como um privilégio em relação ao SUS. Os motivos de insatisfação foram: demoras na marcação de consultas, restrições a tratamentos, procedimentos e internações, e a instalação de espaços público-privados comuns de atenção.

Do ponto de vista metodológico, a literatura oferece distintas perspectivas na abordagem da satisfação. Parasuraman, Zeithaml e Berry (1990) desenvolveram uma das metodologias mais conhecidas para avaliar serviços privados de diferentes naturezas, por meio da avaliação de cinco dimensões do atendimento: agilidade, confiabilidade, empatia, segurança e tangibilidade. Donabedian (1990; 1984) utilizou a categoria aceitabilidade - que significa o grau de conformidade dos serviços oferecidos em relação às expectativas e aspirações dos pacientes e seus familiares.

As propostas destes dois últimos autores têm semelhança conceitual, ao se relacionarem às expectativas que podem ter sido atingidas ou não. Além disso, a dimensão da aceitabilidade contempla elementos tais como: condições de acessibilidade ao serviço, relação médico-paciente, adequação das dependências e instalações, preferências em relação aos efeitos e custos do tratamento e tudo aquilo que o paciente considera justo ou equânime. Estas variáveis podem influenciar de forma mais direta na definição e avaliação da qualidade dos serviços de saúde.

Interessou, para este estudo, a visão de Pascoe (1983) da satisfação do paciente, considerada como uma avaliação da atenção recebida. Esta avaliação é uma comparação de características de destaque da experiência de cuidados de saúde dos indivíduos com um padrão subjetivo, implicando, portanto, atividades psicológicas (no campo perceptual) de ordem cognitiva e afetiva, engajadas em um processo comparativo entre a experiência vivida e critérios subjetivos do usuário (Trad et al., 2002).

O padrão subjetivo usado pelos indivíduos para julgar o cuidado de saúde experimentado pode ser uma, ou a combinação das seguintes dimensões: um ideal subjetivo de atenção, uma percepção subjetiva ou uma noção de atenção merecida, uma média da experiência passada em situações similares ou algum nível subjetivo de qualidade minimamente aceito (Aharony, Strasse, 1993).

Aharony e Strasse (1993) apontam algumas questões metodológicas importantes, advindas de vários problemas conceituais e operacionais, quando se pretende mensurar a satisfação, como, por exemplo: em que momento do atendimento o usuário deve ser abordado; que tipo de pergunta e de escala utilizar, e o que exatamente avaliar.

Frequentemente, as críticas às pesquisas de satisfação recaem sobre o aspecto subjetivo da categoria "satisfação", que sofre influência de grande variedade de elementos determinantes, tais como o grau 
de expectativa e exigências individuais em relação ao atendimento e características individuais do paciente, como idade, gênero, classe social e estado psicológico (Sitzia, Wood, 1997).

Explicações sociopsicológicas têm sugerido que os níveis de satisfação são moldados por diferenças entre as expectativas dos pacientes sobre o serviço e a atenção recebida (Atkinson, 1993). Entretanto, pesquisas recentes têm demonstrado que a expectativa é um conceito deveras complexo, assim como a ausência dela, como, por exemplo, quando há possibilidade de os pacientes terem aprendido a diminuir as suas expectativas quanto à atenção oferecida, ou quando um serviço que tenha recebido uma boa avaliação for resultado de uma baixa capacidade crítica dos usuários, ou o contrário (Aharony, Strasse, 1993).

Calnan (1988) sugere que uma aproximação mais frutífera seria examinar as razões ou motivos por busca de cuidados ao invés de explorar expectativas.

Outra questão presente no debate diz respeito a que os estudos de avaliação da satisfação do usuário têm focado, de uma maneira geral, a medida do nível de satisfação com o serviço prestado, sem um esforço de contextualização cultural. São utilizados escalas e questionários que abordam um sem número de questões, incluindo estrutura, funcionamento e avaliação do serviço de saúde pelo usuário (Williams, 1994; Atkinson, 1993). São comuns perguntas sobre satisfação ou insatisfação, fechadas e do tipo dicotômicas, que são relativas à experiência do usuário, mas excluem o exame das crenças, modos de vida e concepções do processo saúde-doença, elementos que reconhecidamente exercem influência sobre as formas de utilização dos serviços.

Com fins de superar essas limitações, novos caminhos foram trilhados e o desenvolvimento de aproximações qualitativas de investigação, para melhor investigar aspectos subjetivos de usuários, foi incorporado (Trad et al., 2002; Aharony, Strasse, 1993).

Em 2000, a Organização Mundial de Saúde (OMS) introduziu o conceito de "responsividade" dos sistemas de saúde nas pesquisas de avaliação em saúde, para se referir à satisfação de aspectos nãomédicos do cuidado, como acesso e utilização dos serviços (OMS, 2000). Este conceito e as metodologias que incorporam a visão do usuário são considerados como parte de um paradigma no qual se reafirmam princípios relativos a direitos individuais e de cidadania, tais como expressos nos conceitos de humanização e direitos do paciente (Vaitsman, Andrade, 2005).

\section{Metodologia}

Consistiu em um estudo de tipo qualitativo construído com base em dados coletados em entrevistas e em fontes secundárias. Foram realizadas entrevistas semiestruturadas com usuários do subsistema de saúde suplementar residentes em Porto Alegre, Florianópolis e Curitiba, portadores de situações de saúde selecionadas na pesquisa original, em quatro linhas de cuidado: câncer de mama, infarto agudo do miocárdio (IAM), parto e alcoolismo. Considerou-se, como linha de cuidado, a facilitação do acesso ao conjunto de serviços ambulatoriais ou hospitalares, aos cuidados médicos ou de outros profissionais de saúde, e as tecnologias de diagnóstico e tratamento capazes de contribuir para a integralidade do cuidado que as pessoas necessitam (Brasil, 2005).

Foi utilizada uma amostra de dez a 15 entrevistas por situação marcadora e por cidade, totalizando 131 entrevistas nas três capitais. Utilizou-se, como critério de suficiência, a saturação dos dados. As instituições escolhidas foram selecionadas por meio de critérios de conveniência e intencionalidade.

Três processos de amostragem utilizados em pesquisa qualitativa foram articulados para esta construção: intencional, de conveniência e "bola de neve". Para o IAM, a amostra foi intencional, com coleta de dados em pacientes internados na principal prestadora de serviços privados na área cardiovascular, na unidade de terapia intensiva de dois hospitais de referência e em pacientes cadastrados no Programa para Hipertensos e Diabéticos, nas instalações de uma Unidade Básica de Saúde. Para o parto, houve seleção por amostragem intencional a partir de mães que levavam seus bebês para consultas de puericultura no âmbito de um centro de atendimento infantil e em uma Unidade Básica de Saúde, e no domicílio de ex-parturientes de uma listagem de nascimentos fornecida pela Secretaria Municipal de Saúde. A seleção para o câncer de mama se deu a partir da lista de associados da Associação Brasileira de Portadores de Câncer, da Associação das Amigas da Mama e do Instituto da 
Mama. Informantes-chave indicavam outros pacientes, caracterizando a técnica de "bola de neve", com entrevistas realizadas nos domicílios. Para o alcoolismo, a seleção foi por intermédio de contatos com grupos de Alcoólicos Anônimos, nos locais de reuniões, e pela técnica de "bola de neve".

Utilizou-se um roteiro semiestruturado para as entrevistas, que indagou sobre a satisfação com os planos e com os serviços de saúde. Para um cômputo geral quantitativo acerca da satisfação referida, utilizou-se uma escala de tipo Likert, para mensurar o grau de satisfação com as seguintes dimensões: totalmente satisfeito, satisfeito, insatisfeito, muito insatisfeito e ignorado.

Para a análise dos dados obtidos nas entrevistas, optou-se pela utilização da análise temática de Bardin (Minayo, 2007). Iniciou-se com a leitura flutuante de cada entrevista e chegou-se a uma visão do conjunto por linha de cuidado nos três municípios, e da categoria pré-selecionada: satisfação considerada com relação ao atendimento e com o plano de saúde. Para o tratamento dos dados foi utilizado o software Nvivo.

\section{Resultados e discussão}

A doença é uma experiência significativa, que, para ser apreendida, necessita que se identifiquem como são vivenciadas as formas de cuidados e quais as expectativas sobre a atenção a ser recebida (Kleinman, 1980). Neste contexto, o estudo buscou identificar a satisfação dos usuários em relação ao plano de saúde utilizado e aos cuidados recebidos nos serviços envolvidos.

De um modo sintético, a satisfação medida no conjunto das linhas resultou em uma avaliação significativamente positiva (Tabela 1).

Observa-se que, nesta modalidade de medida, tanto o atendimento dos serviços conveniados aos planos de saúde quanto os próprios planos receberam graus positivos de avaliação da grande maioria dos entrevistados das três capitais. Vários pontos de insatisfação apareceram, porém, durante as falas e estão descritos em cada linha pesquisada.

Tabela 1. Distribuição do grau de satisfação em quatro linhas de cuidado nas três capitais, $2008(n=131)$

\begin{tabular}{|c|c|c|c|c|c|}
\hline Variáveis & Alcoolismo & Infarto Agudo do Miocárdio & Câncer de mama & Parto & Todas (\%) \\
\hline \multicolumn{6}{|c|}{ Satisfação com o atendimento } \\
\hline Totalmente satisfeito & 15 & 23 & 21 & 25 & $84(64,1)$ \\
\hline Satisfeito & 12 & 8 & 9 & 8 & $37(28,2)$ \\
\hline Insatisfeito & - & 1 & 2 & 1 & $4(3,1)$ \\
\hline Muito insatisfeito & - & - & 1 & 3 & $4(3,1)$ \\
\hline Ignorado & 2 & - & - & - & $2(1,5)$ \\
\hline \multicolumn{6}{|l|}{ Satisfação com o plano } \\
\hline Totalmente satisfeito & 5 & 18 & 21 & 15 & $59(45,5)$ \\
\hline Satisfeito & 20 & 9 & 9 & 19 & $57(43,5)$ \\
\hline Insatisfeito & 3 & 4 & 2 & 2 & $11(8,4)$ \\
\hline Muito insatisfeito & - & 1 & 1 & 1 & $3(2,3)$ \\
\hline Ignorado & 1 & - & - & - & $1(0,8)$ \\
\hline
\end{tabular}

Fonte: Entrevista aos usuários participantes da pesquisa, 2008.

\section{Linha de cuidado cardiovascular, marcador "infarto agudo do Miocárdio (IAM)"}

a) Satisfação com os serviços e com a qualidade da assistência recebida: a satisfação positiva com os serviços foi unânime entre os entrevistados, sendo o aspecto melhor avaliado dos planos de saúde. Nas três capitais, a maioria dos usuários escolheu a alternativa "totalmente satisfeitos" para qualificar a atenção recebida, que se relacionou, sobretudo, à boa atenção profissional recebida na clínica de internação, com destaque para o reconhecimento do diferencial que significa obter atenção de boa qualidade. Foram valorizados: a presteza no atendimento, a qualidade técnica, o tratamento de acordo 
com as necessidades individuais e com as diferentes subjetividades, o acesso aos cuidados necessários e a cobertura oferecida.

\author{
“Totalmente satisfeito. Porque todo o pessoal foi atencioso, competente. A médica que me \\ atendeu no primeiro dia continua me dando cobertura até o fim... [...] aqui na UTI tem \\ plantão. Troca a cada seis horas e todos eles são muito atenciosos". (Cl 01) \\ “Do serviço não posso me queixar. Se pudesse dar mais de dez, eu daria. Tanto na \\ ambulância quanto aqui, na estadia, no tratamento [...]". (F16)
}

Também houve referência de bom tratamento em um hospital do setor público:

"Fui muito bem tratado, peguei uma equipe ótima de serviço lá no HU e o pessoal me deu toda atenção, tanto é que eu só fiquei vinte minutos numa emergência [...]". ( F4)

Os pontos de insatisfação se referiram a queixas relativas a um ambiente hospitalar ruidoso, ao tempo de espera para a internação e ao mau manejo profissional:

"[...] Com o lado profissional eu fiquei totalmente satisfeito, mas sobre o total, não, por causa de uma tremenda algazarra das visitas aqui ao lado". (F13)

"Devia ter mais leitos, mais espaço, pois se espera em fila, perdendo tempo, sofrendo. Faz uma semana que a gente tá esperando leito no ' $\mathrm{X}$ '". (F1)

“Insatisfeito [...] eu fui duas horas da manhã pro hospital, peguei o profissional de plantão, não tinha papel no aparelho: 'vamos fazer exames de enzimas, esse exame é demorado, então você pode ir pra casa e depois voltar'. Quando voltei e viram o resultado, me enfiaram numa maca na mesma hora. Foi interessante, porque eu fiquei num apartamento, apesar do meu plano ser enfermaria [...]". (C11)

b) Satisfação com o plano: a maioria dos usuários relatou grande satisfação com o plano de saúde, valorizando de forma muito positiva a presteza do atendimento, com acesso facilitado e qualidade proporcionada por meio do serviço de saúde contratado. As insatisfações se referiram: à cobertura do plano, inclusive com relação ao acompanhante, às mensalidades consideradas altas, às necessidades eventuais de copagamento, e aos custos crescentes.

"A cobertura é total, mas o plano já mandou dizer que não quer pagar alguma coisa, mas eu já entrei com advogado pra pagar uma ultra-sonografia, pra continuar a parte preventiva, mas tá no plano. Na tabela antiga diz que eu tenho direito, mas alguns hospitais estão usando as tabelas novas, que dizem que eu não tenho direito". (F5)

"O plano atendeu minhas necessidades imediatas. A reclamação é que nos primeiros dias o acompanhante não recebeu alimentação, não tinha direito... E cinco dias depois disseram que receberam uma informação do plano corrigindo esse erro". (F 11)

Com resultados semelhantes, Milan e Trez (2007), em estudo sobre a satisfação de beneficiários de planos de saúde, mostraram que as razões para a satisfação com os planos são as certezas de acesso ao tratamento e a hotelaria e privacidade.

Em Florianópolis, e em menor volume em Curitiba, as maiores insatisfações relatadas estiveram relacionadas às dificuldades de acesso a exames complementares (liberação, autorização) e à cobertura oferecida pelo plano de saúde. A autorização para prestação de serviços entre cooperadas de diferentes estados foi alvo de críticas: 


\footnotetext{
"Um pouco demorado [...] a liberação... os procedimentos mais caros, como cateterismo, eles demoram pra liberar ...". (F1)

"A autorização do meu plano acontece por Tocantins! E não fazem liberação por telefone! Liberam pelo sistema da 'y' e só na unidade da rua tal. É uma dificuldade grande!". (CI 10)
}

Com relação às insatisfações com custos crescentes e copagamento, houve regulação recente da Agência Nacional de Saúde Suplementar (ANS, 2009) sobre a matéria, no sentido de responder a questionamentos com este teor. Gerschman et al. (2007), ao analisarem a satisfação dos beneficiários de planos de saúde, encontraram que o padrão da prestação de serviços está associado ao preço do plano e à qualidade do hospital a que está vinculado. Independentes de serem planos novos ou antigos, os planos de categorias mais altas, de hospitais bem estruturados, prestam um serviço de melhor qualidade. Da mesma forma, planos de categorias mais baixas, de hospitais pouco estruturados, oferecem um serviço pior. Aqui também parece ser esta a explicação para as pessoas que tiveram maior ou menor insatisfação com os diferentes planos, independentemente da operadora.

De forma semelhante, os motivos de insatisfação encontrados parecem conformar um padrão de respostas encontradas em vários estudos no país: demora na marcação de consultas; restrição ao tratamento de doenças cardíacas e outras; restrição no número de consultas e exames e tempo de internação e de UTI. Também nesta pesquisa, as regras ou a falta delas para os reajustes por idade e a restrita abrangência geográfica de alguns planos eram motivos de descontentamento com o plano de saúde.

Destacou-se, entre os usuários das três capitais, uma grande preocupação com a questão dos medicamentos, e há uma tendência a responsabilizar o setor público pelo fornecimento deles ou a desqualificar sua qualidade.

“Medicação graças a Deus eu tô pegando lá no Centro de Saúde, tem poucos que eu compro. Mas o cardiologista não quer que eu continue com esse, disse que não é a mesma coisa". (PI 63)

\section{Satisfação na linha de cuidado materno-infantil: marcador "parto"}

a) Satisfação com os serviços - a maioria das mães entrevistadas valorizou: a presteza e atenção no atendimento, o tratamento de acordo com as necessidades individuais e subjetivas, o acesso à atenção disponível, quando necessário, e a cobertura oferecida. A maioria referiu total satisfação com o atendimento prestado pelos profissionais nas maternidades onde foram atendidas, tanto públicas como privadas.

\footnotetext{
"Acho que foi excelente, inclusive as próprias médicas. Recebi uma apostila falando sobre os diferentes tipos de parto, sobre os tipos de anestésicos e os procedimentos em relação ao pré e o pós-cirúrgico". (CO 05)
}

As insatisfações se relacionaram à qualidade do atendimento prestado pelos profissionais de saúde e por recepcionistas, desde o internamento até a alta. As principais queixas foram relativas à falta de informação sobre as condições da mulher durante o parto e às condições do bebê.

“Faltou bastante da parte da enfermagem, sabe? Me colocaram lá, era prematuro. Acho que tinham que ter conversado mais, porque eu chorava de dor e de medo, porque eu achava que o nenê não ia sobreviver [...]". (CO 06)

b) Satisfação com o plano: de uma forma geral, a maioria das usuárias referiu satisfação com o plano de saúde. 
"Satisfeita [...] às vezes, a gente não conseguia a consulta no dia que precisava, mas a minha doutora sempre fazia encaixe... O plano em si até que tá ruim, mas ela dava um jeito e eu era atendida". (CO 08)

Também aqui as insatisfações ficaram por conta: das falhas na cobertura do plano, das necessidades eventuais de copagamento, dos custos crescentes e, sobretudo, das condições de estrutura física e humana das maternidades conveniadas aos planos de saúde.

“[...] Imagina você, pós-cirurgia, num quarto onde fica 24 horas cheirando a esgoto, e ninguém toma nenhuma iniciativa?". (CO 04)

“Embora eu tenha ' $y$ ', eu tive que pagar tudo particular, menos as consultas. Eu achava que ' $y$ ' fosse tudo igual, em todo Brasil e não é [...] eles não quiseram comprar a carência do parto, compravam a carência de consulta de outras coisas [...]". (CO3)

Embora não tenham sido foco central nesta pesquisa, os aspectos relacionados à humanização do atendimento no evento do parto ganharam evidência nas respostas das entrevistadas, demonstrando a importância da assistência humanizada e de uma rede de atenção mais estruturada, com equipe multiprofissional ampliada.

"Acho que tinha que ter psicóloga pra conversar comigo, pra me acalmar. As enfermeiras lá, são muito frias... Me senti muito sozinha, com dor e com medo ao mesmo tempo". (CO 06)

"Só o que eu queria agora era um acompanhamento de psicoterapia, só que psicólogo mesmo, não cobre". (F04)

\section{Satisfação na linha de cuidado oncologia: marcador "câncer de mama"}

a) Satisfação com o atendimento: Em geral a satisfação foi grande, o atendimento considerado bom, individualizado, respondendo às expectativas de escuta e tratamento carinhoso e compreensivo. A competência profissional e o fato de terem apresentado um desfecho favorável justificaram também, em alguns casos, a satisfação.

“... eu acho que o que tinha que ser feito, foi feito. O que tinha que ser falado, falaram [...]... fico muito satisfeita por ter passado por profissionais tão competentes". (CM 06)

“Eles são realmente queridos com a gente, não é um atendimento profissional, é um atendimento pessoal". (FM 04)

Insatisfações ocorreram quando o atendimento não foi individualizado ou sem atenção especial à paciente.

\footnotetext{
“No atendimento profissional eu acho que [falta] mais humanização sabe? [...] uma pessoa que tá passando mal quer falar, falar. E as pessoas [profissionais] não têm tempo... Não foram ruins, mas se passassem por orientações, ou trabalhassem uma semana e dois dias folga, ou tivessem um lazer diferente, seriam mais humanos". (CM 04)

"[...] quando eu perguntei quando é que vinha o exame dos nódulos, uma pessoa disse "uns doze dias", e eu disse:" que horror, eu vou ficar doze dias nessa angústia?" "ah tem"', eu quase morri". (PM 01)
}

Segundo Gimenes e Queiroz (1988), os profissionais que lidam com pacientes com câncer de mama devem estar capacitados a trabalharem os anseios dessas pessoas. Rossi e Santos (2003) ressaltaram a 
importância de uma boa relação médico-paciente nestes casos, impactando, inclusive, a aderência ao tratamento por parte da paciente.

Um ambiente de tratamento desagradável também foi referido como fator associado à insatisfação no atendimento.

“Tratar de câncer já é uma coisa pesada. Se não tiver um lugar agradável, com pessoas que te atendam com carinho... Então, eu acho que deve mudar a aparência física do local". (CM 06)

b) Satisfação com o plano: em geral houve grande satisfação com o plano de saúde. Destacaram-se as experiências com um fluxo rápido no atendimento por parte da operadora e, também, quando a cobertura do plano correspondia às necessidades do beneficiário.

"[Satisfeita] porque cobriu tudo que eu precisava, não tive de dispender nada, foi excelente". (PM 09)

Algumas entrevistadas indicaram a importância da inclusão de outros profissionais, além do médico e enfermagem, durante o tratamento da doença:

“Eu acho que seria muito bom um assistente social. Talvez até tenha, mas eu acho que tinha que ser um pouco mais presente, acompanhando o tratamento...". (CM 10)

“Também acho que devem oferecer outros profissionais, como o fisioterapeuta e o psicólogo...". (CM 14)

No primeiro ano após a realização da cirurgia, as mulheres têm dificuldade para desenvolver atividades profissionais e esforços para execução de tarefas relacionadas ao seu dia a dia pessoal, por perda de força muscular e de amplitude de movimento (Shimozuma et al., 1999). Além disso, há o impacto, muitas vezes avassalador, que o diagnóstico e o tratamento da doença provocam, que deve ser abordado profissionalmente. Fica clara a necessidade de uma abordagem multi e interdisciplinar nas práticas assistenciais.

Aspectos burocráticos na liberação de exames também foram referidos:

"Alguns exames tem que levar na sede da 'y' pra autorizar. Leva de 24 a 48 horas, tem que passar pela auditoria, fica nessa expectativa por 48 horas!". (PM 05)

"A única coisa que eu não concordo é que eles não têm uma sede aqui no centro, eu preciso dos ônibus pra ir lá num bairro, pra liberar um exame mais caro". (CM 07)

A dificuldade de acesso aos medicamentos para tratamento representou grande frustração para as beneficiárias, havendo necessidade de recorrerem ao SUS para seguirem com o tratamento. Esta parece ser uma das principais limitações dos planos de saúde quanto ao tratamento do câncer de mama.

“... Minha médica falou pra ir no SUS da Santa Casa para ver se consigo os remédios, porque eles são muito caros, né!?". (PM 02)

“O convênio tá me pagando praticamente tudo, só não me restitui, por exemplo, medicamentos para os efeitos colaterais, insônia, dores no corpo, irritação, problemas no estômago...". (FM 08)

A partir de estudo de base populacional, Lima-Costa (2002) descreveu que o acesso aos medicamentos configura-se como importante problema entre beneficiários de planos de saúde. Dados da Pesquisa Nacional por Amostra de Domicílio e da Pesquisa de Orçamentos Familiares indicaram que os 
maiores gastos das famílias com saúde são as mensalidades com os planos de saúde e a compra de medicamentos (Silveira, Osório, Piola, 2002).

Outra preocupação das pacientes foi com a rede de médicos conveniados com os planos de saúde. Em diversos momentos, as beneficiárias precisaram abrir mão do seu médico de confiança, ou que as acompanhava há muitos anos, para procurar outro, conveniado ao plano. Além disso, há apreensão sobre a permanência dos credenciados no futuro. Numa avaliação quantitativa da satisfação de beneficiários de planos de saúde em relação à sua operadora, Milan e Trez (2007) identificaram como fatores associados: (i) a qualidade do atendimento recebido pelo paciente; (ii) os médicos que têm à disposição para a marcação de consultas, valorizando a confiança e a segurança na hora de escolher ou manter a preferência por um profissional; (iii) o preço do plano, e (iv) a conveniência e facilidade para marcação de consultas e exames, a oferta de serviços complementares e a cobertura do plano.

"A única coisa que eu fico apreensiva é que o médico é conveniado e meu medo é de um dia ele se descredenciar, porque é um plano que não paga muito. Já passei por uns quatro, cinco médicos, cada vez que eu marcava uma consulta, ele já tinha se descredenciado". (CM 11)

"Gostaria que houvesse mais profissionais médicos especialistas, deveria ter mais opção...". (PM 01)

\section{Satisfação na linha de cuidado saúde mental: marcador "alcoolismo"}

a) Com o atendimento recebido: As entrevistas mostraram grande insatisfação com o atendimento formal. Severas críticas relativas à assistência médica e ao aspecto comercial de algumas clínicas foram explicitadas. Simultaneamente, há um certo consenso de que a "decisão" pessoal de manter a abstinência está intimamente ligada ao sucesso dos tratamentos.

"O tratamento foi fantástico, o atendimento que eles deram e a forma que foi conduzido. Era muita reunião, troca de idéias, e o que eu fiz ao sair de lá, foi ir pro grupo (de AA) onde estou até hoje". (FA 01)

"A minha insatisfação é em função do atendimento psiquiátrico. [...] Eu já tinha feito os meus trinta dias. Quinze de desintoxicação, e os outros pra terminar o tratamento, que era ficar lá amassando barrinho, que para algumas pessoas pode ajudar em alguma coisa e para outras é totalmente inócuo". (FA 02)

Há uma diversidade de percepções sobre os diferentes atendimentos recebidos, mas destaca-se a crítica pelo despreparo profissional para o trabalho com este tipo de dependente, gerando insatisfações com a atenção. Observa-se grande valorização dos tratamentos informais, tais como de comunidades terapêuticas e AA.

“[...] as comunidades terapêuticas são tão úteis quanto uma clínica, porque a clínica tem toda a estrutura, mas dependendo do caso que se encontra a dependência química, trinta dias é pouco...". (CA05)

"AA é muito bom... Eu venho de uma terra muito fria e para se esquentar só tem duas formas, usando um casaco de pele e acendendo uma fogueira. O casaco só vai esquentar quem o veste, já a fogueira pode esquentar a si e a quem chegar perto. Então eu vejo todos os meus companheiros de AA como uma fogueira, não é egoísta, é só chegar perto. Eu sei que ele torce por mim e eu torço por ele também. Eu não sei nada sobre eles, o importante é estar lá". (FA 08)

As comunidades terapêuticas foram identificadas como lugares que proporcionam um tempo maior de afastamento do cotidiano externo, percebido como necessário, e que não ocorre devido ao prazo das 
internações dos planos de saúde. A satisfação se relaciona a um redimensionamento de valores que se aproximam a uma integralidade que vai muito além da solução clínica alcançada na biomedicina.

b) Satisfação com o plano: insatisfações com a cobertura do plano, especialmente nos limites para custeio do atendimento necessário - com destaque para as dificuldades de reconhecimento do alcoolismo como doença pelos profissionais -, foram apontadas nesta linha de cuidado. Além disso, insatisfações quanto aos custos com o pagamento da mensalidade do plano e de situações que demandam mais tempo de tratamento ou copagamento (pelo plano e pelo usuário) também foram relatadas. Os poucos depoimentos de satisfação plena se referiram aos aspectos de cobertura total do plano, e não da qualidade do atendimento.

$\mathrm{Na}$ ausência de uma ampla cobertura, a tendência foi a utilização do SUS ou pagamento do próprio bolso, sobretudo nas consultas psiquiátricas.

“... não totalmente satisfeito, com uma franquia de mil e quinhentos reais... e pelo número de consultas, eu acho injusto dizerem quantas eu posso consultar...". (PA 03)

“... Insatisfeito com os planos; o profissional que nos acompanha (internações), por não ser credenciado, não pode nos atender. E hoje os planos estão restritos a uma ou duas instituições hospitalares na cidade. Outra situação é a falta de credenciamento de outros profissionais, como fisioterapeuta, psicólogo...". (CA 04)

\section{Conclusões}

Os estudos de satisfação, apesar de oferecerem informação limitada sobre seus determinantes (Ricketts, Kirshbaum, 1994), apontam a possibilidade de se ampliar a compreensão sobre as vivências e as expectativas dos usuários em relação ao modo como os serviços de saúde - e, nesse particular, os serviços conveniados a planos de saúde - estão ou devem estar organizados para solucionar problemas específicos e atender às suas necessidades.

Ao compararmos as linhas de cuidado analisadas, algumas tendências acerca da satisfação com o atendimento e planos de saúde, presentes nos diferentes arranjos de utilização dos sistemas de cuidados em cada uma delas, puderam ser apontadas. Isto foi facilitado pela utilização da entrevista semiestruturada, que supriu as lacunas que um questionário fechado poderia conferir ao enfoque estudado.

Foi alta a satisfação referida pelos usuários com relação aos prestadores do sistema profissional e aos planos de saúde nos atendimentos recebidos no caso do IAM, do câncer de mama e durante o parto. Ter acesso rápido aos recursos que interpretaram como sendo de qualidade técnica adequada ou com maior aporte tecnológico é o aspecto que mais sobressaiu nessa avaliação. Especialmente no caso do IAM, esta última característica foi determinante para a alta satisfação referida. Os pontos de insatisfação diziam respeito a dificuldades de informações e com as instalações.

Houve uma satisfação mais baixa no caso do alcoolismo, quando comparado às demais situações. A ênfase dos aspectos negativos recaiu sobre o elevado custo, sobretudo nos casos onde o período de internação era grande, havendo uma satisfação maior apenas nos planos com cobertura total. A falta de vagas, tanto nos hospitais psiquiátricos, quanto nas clínicas e nos hospitais gerais que atendem casos de alcoolismo, foi um elemento presente nas três capitais. Encontrou-se um amplo espectro de insatisfações relativas à qualidade da assistência em todos os níveis da atenção ao alcoolismo, sobretudo quanto ao despreparo do profissional médico para o trabalho com dependentes, e críticas relativas ao caráter lucrativo dos planos de saúde. O sistema informal, especialmente a organização AA, possui uma avaliação bastante positiva.

A necessidade da inclusão e maior disponibilização, pelos planos de saúde, de assistentes sociais, fisioterapeutas, nutricionistas e psicólogos, por exemplo, também foi bastante evidenciada pelos usuários. Além disso, o desejo de ampliação da rede de médicos beneficiados foi descrito por muitos usuários, sobretudo para consultas com especialistas. 
Quanto aos planos, cujo índice de satisfação é alto, porém menor do que em relação aos prestadores de serviços de saúde, as insatisfações nas quatro situações de saúde ficaram em torno do custo crescente, das necessidades de copagamento, das deficiências de coberturas e dos impasses burocráticos para autorizações de procedimentos. Talvez isto possa, em parte, ser explicado pela ambiguidade referente à existência de duas lógicas decorrentes da segmentação do sistema brasileiro: a do cidadão e a do consumidor. Embora inserido numa estrutura contratual de mercado, o imaginário dos usuários é permeado por aspirações de uma cobertura universal.

Há uma discussão a ser feita quanto ao viés de valorização positiva das respostas obtidas diante de certas situações vitais. Assim, no caso do parto, quando as mulheres eram estimuladas a falarem mais acerca de seus desejos e expectativas em relação ao plano e aos serviços, muitos aspectos relacionados à humanização (paciência, apoio, carinho) vieram à tona. Também, no caso do câncer de mama, apesar de, na pergunta objetiva, as pacientes referirem majoritariamente satisfação com o plano, expressiva parte delas mostrava ressalvas. Referiam à necessidade da cobertura possuir uma maior diversidade de profissionais não-médicos, uma vez que o tratamento requer uma integração entre diversas áreas do conhecimento.

O desenvolvimento de novos referenciais e instrumentos que possibilitem uma visão sobre a satisfação dos usuários e de sua rede de relações pode se revelar especialmente útil para a atenção à saúde atualmente. Aspectos da satisfação dos usuários podem ser manifestos com mais amplitude, à semelhança deste estudo, que mostrou que o caráter "tecnológico" característico da linha cardiovascular é fator determinante de satisfação, mas também a necessidade de realização de práticas de promoção ou prevenção efetivas. Na mesma linha, a oncologia representa, juntamente com o parto, excelente situação para acompanhamento das ações de humanização e da presença de equipe multiprofissional de saúde no acompanhamento de pacientes. A saúde mental oferece a possibilidade de se reconhecerem os limites e a capacidade de resposta da rede de assistência biomédica, assim como da ampliação do diálogo entre o sistema profissional e o informal.

As narrativas das experiências dos usuários permitiram identificar os múltiplos aspectos implicados na qualidade da atenção recebida, que devem ser levados em conta na implementação de políticas e programas que se relacionam com a promoção da saúde, com a efetividade e a melhoria estrutural da rede de serviços de saúde. Esses resultados apontam, ainda, a necessidade do desenvolvimento de atitudes e ações que possibilitem construir a integralidade da atenção e do cuidado nas linhas estudadas, evidenciando o papel da humanização da assistência, o desenvolvimento de relações coordenadas entre os sistemas público, privado e informal de cuidados, além dos aspectos econômicos e arranjos técnico-assistenciais presentes na atenção à saúde.

\section{Colaboradores}

Maristela Chito Sisson e Maria Conceição de Oliveira participaram do delineamento da pesquisa, coleta de dados, e foram as responsáveis pela redação do artigo. Eleonor Minho Conill e Denise Pires coordenaram o estudo, participaram do delineamento da pesquisa, coleta de dados, e contribuíram na redação do artigo. Antonio Fernando Boing e Hosanna Pattrig Fertonani participaram do delineamento da pesquisa, coleta de dados, e contribuíram na redação do manuscrito.

\section{Agradecimentos}

Os autores agradecem à Agência Nacional de Saúde Suplementar que, com a política de fomento à pesquisa por meio do estabelecimento de centros colaboradores regionais, garantiu os subsídios necessários à realização do estudo. 


\section{Referências}

AGÊNCIA NACIONAL DE SAÚDE SUPLEMENTAR. Resolução Normativa n.195, de 14 de julho de 2009. Disponível em: <http://www.ans.gov.br/portal/site/legislacao/ legislacao_integra.asp?id=1856\&id_original=0>. Acesso em: 20 maio 2010.

AHARONY, L.; STRASSE, S. Patient satisfaction: what we know about and what we need to explore. Med. Care Rev., v.50, n.1, p.49-79, 1993.

ATKINSON, S.J. Anthropology in research on the quality of health services. Cad. Saude Publica, v.9, n.3, p.283-99, 1993.

BERGER, T. et al. The organization of quality assurance. Int. J. Qual. Health Care, v.1, n.2-3, p.111-23, 1989.

BRASIL. Agência Nacional de Saúde Suplementar. Programa de Qualificação da Saúde Suplementar. Qualificação da saúde suplementar: nova perspectiva no processo de regulação. Rio de Janeiro: ANS, 2005. 1 cd-rom.

CALNAN, M. Towards a conceptual framework of lay evaluation of health care. Soc. Sci. Med., v.27, n.9, p.927-33, 1988.

CONILL, E.M. et al. O mix público-privado na utilização de serviços de saúde: um estudo dos itinerários terapêuticos de beneficiários do segmento de saúde suplementar brasileiro. Cienc. Saude Colet., v.13, n.5, p.1501-10, 2008.

DE SILVA, A. A framework for measuring responsiveness. Geneva: World Health Organization, 1999. GPE Discussion Paper Series: n.32.

DONABEDIAN, A. La calidad de la atención medica: definición, método e evaluación. México: La Prensa Médica Mexicana, 1984

Exploration in quality assessment and monitoring: the definition of quality and approaches to its assessment. Ann Arbor: University of Michigan, 1980.

FAVARO P.; FERRIS L.E. Program evaluation with limited fiscal and human resources. In: LOVE, A.J. (Ed.). Evaluation methods sourcebook. Ottawa: Société Canadienne d'Evaluation, 1991. p.4-25.

FITZPATRICK, R. Surveys of patient satisfaction: important general considerations. Brit. Med. J., v.302, p.88-90, 1991.

GERSCHMAN, S. et al. Estudo de satisfação dos beneficiários de planos de saúde de hospitais filantrópicos. Cienc. Saude Colet., v.12, n.2, p.487-500, 2007.

GIMENES, M.; QUEIROZ, E. As diferentes fases de enfrentamento durante o primeiro ano após a mastectomia. In: GIMENES, M. (Org.). A mulher e o câncer. Campinas: Editorial Psy, 1997. p.173-95.

KLEINMAN, A. Patients and healers in the context of culture: an exploration of the borderland between, antropology, medicine and psychiatric. London: University of Califórina Press, 1980.

LIMA-COSTA, M.F.F. Projeto Bambuí: plano privado de saúde e utilização de serviços médicos pela população idosa. Cad. Saude Publica, v.18, n.1, p.177-86, 2002.

LINDER-PELZ, S. Toward a theory of patient satisfaction. Soc. Sci. Med., v.16, n.5, p.57782, 1982.

MILAN, S.G.; TREZ, G. Pesquisa de satisfação: um modelo para planos de saúde. RAE Eletrôn., v.4, n.2, p.1-21, 2005. Disponível em: <http://www.rae.com.br>. Acesso em: 30 abr. 2007.

MINAYO, M.C.S. O desafio do conhecimento. 10.ed. São Paulo: Hucitec, 2007.

ORGANIZAÇÃO MUNDIAL DE SAÚDE. The World Health Report - Health Systems: improving performance. Disponível em: <http://www.who.int/whr/en/>. Acesso em: 30 abr. 2007. 
PARASURAMAN, A.; ZEITHAML, V.A.; BERRY, L. Measuring customer service satisfaction. 1990. Disponível em: <http://www.imt.za/qrater/gaps.html>. Acesso em: 13 mar 2007.

PARKER, G. et al. The development of a patient satisfaction measure for psychiatric outpatients. Aust. N. Z. J. Psychiatry, v.30, n.3, p.343-9, 1996.

PASCOE, G.C. Patient satisfaction in primary health care: a literature review andanalysis. Eval. Program Plann., v.6, n.3, p.185-210, 1983.

RICKETS, T.; KIRSHBAUM, M.N. Helpfulness of mental health day care: client and staff views. J. Adv. Nurs., v.20, n.2, p.297-306, 1994.

ROSSI, L.; SANTOS, M.A. Repercussões psicológicas do adoecimento e tratamento em mulheres acometidas pelo câncer de mama. Psicol. Cienc., v.23, n.4, p.32-41, 2003.

SHIMOZUMA, K. et al. Quality of life in the first year after breast cancer surgery: rehabilitation needs and patterns of recovery. Breast Cancer Res. Treat., v.56, n.1, p.4557, 1999.

SILVA JUNIOR, A. Experiências de avaliação do setor suplementar de saúde: contribuições da integralidade. Cienc. Saude Colet., v.13, n.5, p.1489-500, 2008.

SILVEIRA, F.G.; OSÓRIO, R.G.; PIOLA, S.F. Os gastos das famílias com saúde. Cienc. Saude Colet., v.7, n.4, p.719-31, 2002.

SITZIA, J.; WOOD, N. Patient satisfaction: a review of issues and concepts. Soc. Sci. Med., v.45, n.12, p.1829-43, 1997.

TRAD, L.A.B. et al. O estudo etnográfico da satisfação do usuário do Programa de Saúde da Família (PSF) na Bahia. Cienc. Saude Colet., v.7, n.3, p.581-9, 2002.

VAITSMAN, J.; ANDRADE, G.R.B. Satisfação e responsividade: formas de medir a qualidade e a humanização da assistência à saúde. Cienc. Saude Colet., v.10, n.3, p.599613, 2005.

VUORI, H. Patient satisfaction - an attribute or indicator of the quality of care? Qual. Rev. Bull., v.13, n.3, p.106-8, 1987.

WILLIAMS, B. Patient satisfaction: a valid concept? Soc. Sci. Med., v.38, n.4, p.509-16, 1994.

SISSON, M.C. et al.Satisfacción de los usuarios en la utilización de servicios públicos y privados de salud en itinerarios terapéuticos en el sur de Brasil. Interface - Comunic., Saude, Educ., v.15, n.36, p.123-36, jan./mar. 2011.

El estudio trata de la satisfacción de las personas en los itinerarios recorridos durante la utilización de los servicios de salud públicos y privados en tres capitales del sur de Brasil frente a situaciones de salud-enfermedad-cuidados. Trabajando con un planteamiento cualitativo se han entrevistado 131 usuarios de planes de salud que han recibido cuidados en infarto agudo del miocardio, alcoholismo, cáncer de mama y parto. En itinerarios dotados de numerosas trayectorias y arreglos entre sub-sistemas de servicios, sobre todo en campo de la salud mental y de la oncología, la categoría "satisfacción" ha mostrado buena evaluación en el infarto agudo del miocardio, cáncer de mama y parto, por la presencia de acceso rápido a recursos técnicos adecuados. Las insatisfacciones se refieren a dificultades en las informaciones, instalaciones, costes, coberturas y autorizaciones de procedimientos. El estudio ha mostrado la importancia de considerar la pluralidad de los sistemas de cuidados y la subjetividad del juicio de valor en la implementación de políticas y programas de salud.

Palabras clave: Evaluación en salud. Satisfacción del paciente. Servicios de salud. Salud complementaria. 